\title{
Perceptions and attitudes towards gym use and physical activity in young men with haemophilia
}

Luke Pembroke, Laurence Woollard

Introduction: A growing body of evidence supports the recommendation of both physiotherapy and physical activity in people with haemophilia. Physical benefits include increasing strength and flexibility and reducing the risks of osteoporosis, arthropathy, and intramuscular and joint bleeds; social benefits have also been observed. However, anecdotal evidence suggests that people with haemophilia may still be averse to engaging with physical activity due to fears of causing bleeding, joint pain and joint damage. Methodology: Qualitative interviews were conducted with young men with haemophilia treated at comprehensive care centres in London, to explore and identify the reasons behind risk-averse behaviours towards exercise and physical activity. The interview questions were designed to prompt discussion and capture opinions relating to participants' physical activity and gym membership/ use, and the degree to which their haemophilia impacts on both. Results: Ten participants were interviewed. Preferred activities were variable, with five participants describing themselves as very physically active, three moderately active, and two reporting little physical activity; four described themselves as 'not gymconfident'. Seven participants described themselves as

LUKE PEMBROKE

Haemnet, London, UK. Email: luke@haemnet.com

LAURENCE WOOLLARD

On The Pulse Consultancy, Cambridge, UK

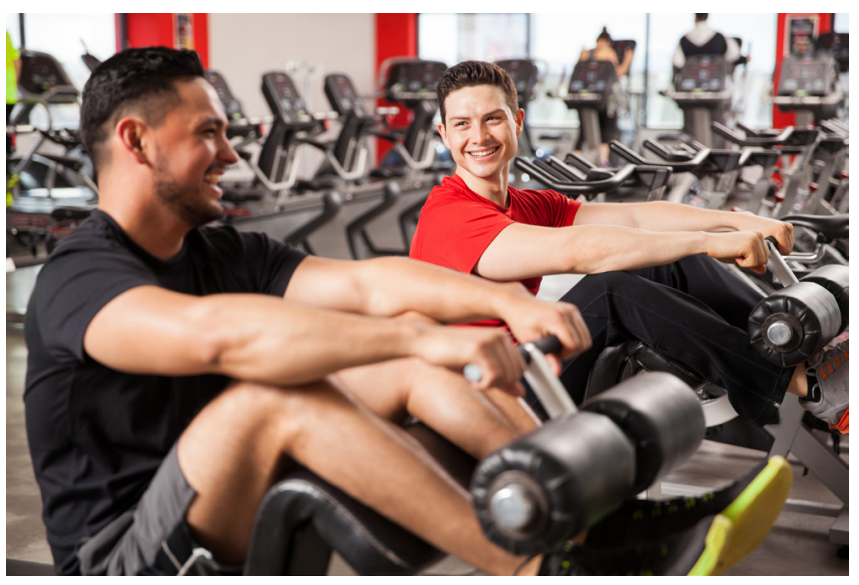

A recent study exploring physical activity among young men with haemophilia highlights their keenness to incorporate gym use into their personal fitness regimens and the importance of a tailored approach to support this

highly or moderately motivated to undertake physical activity, with motivations including weight loss and getting fit for summer holidays. However, there was some anxiety around weight-bearing exercise due to the fear of pain or injury. All participants had been exposed to personal trainers (PTs) and recognised the importance of being properly introduced to training equipment but felt that PTs were too expensive for them. The majority of participants reported sportsrelated injuries and self-perceived limitations on activity due to their personal/individual experience of living with haemophlia. Physiotherapists were often the first point of contact for advice and support on safe physical activity. All participants recognised the benefits of

This is an Open Access article distributed under the terms of the Creative Commons Attribution-NonCommercial-NoDerivs License (https://creativecommons.org/licenses/by-nc-nd/3.0/) which permits use and distribution in any medium, provided the original work is properly cited, the use is non-commercial, and no modifications or adaptations are made. Copyright is retained by the authors. 
physical activity and had been encouraged in this by their physiotherapists. Conclusion: Young men with haemophilia are keen to use the gym as part of their personal fitness regimens. The ongoing safety concerns of health care professionals warrants further research.

Keywords: Haemophilia, gym use, physical activity, perception and attitudes

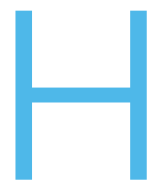

istorically, physical activity was

contraindicated in people with haemophilia due to limited access to treatment for bleeding episodes and the perception that harm would be caused ${ }^{[1]}$. Despite dramatic improvements in the availability of treatment, such as primary prophylaxis for those with severe haemophilia ${ }^{[2]}$, together with a recognition that exercise is beneficial ${ }^{[3]}$, the perception amongst some clinicians and people with haemophilia that physical activity can be harmful still persists. The fear of adverse consequences of injury result in risk aversion and a reluctance to engage in physical activity.

Today, for people with haemophilia, physiotherapy and physical activity are recommended where risks can be managed ${ }^{[4,5]}$. This change in approach is supported by a growing body of evidence that physical activity in people with haemophilia increases strength and flexibility, decreases body fat, and reduces the risk of metabolic and cardiovascular disease, osteoporosis, arthropathy and intramuscular or joint bleeds $[3,6,7]$. Physical activity has been shown to improve physical performance and health-related quality of life ${ }^{[8-11]}$. However, the optimal dose-response for exercise prescription for people with haemophilia has not been well addressed ${ }^{[12]}$. A recent Cochrane review concluded that most exercise interventions for people with haemophilia produced improvement in outcomes such as pain, range of motion, strength and walking tolerance but that the quality of the evidence was poor ${ }^{[13]}$.

Pilot study data support the importance of resistance training in people with haemophilia for increasing muscle strength and decreasing the frequency and severity of bleeding episodes and associated pain ${ }^{[14]}$. However, in a subsequent observational study of young people with haemophilia aged 12-25, the same group found that those who exercised strenuously had a higher proportion of trauma-related bleeds ${ }^{[15]}$.

London haemophilia services work with approximately one third of the UK's haemophilia population and includes a cohort of around 150 young men aged $18-25$ years. This group consists of a large number of young men who, despite receiving intensive prophylaxis and home care programmes, have damaged ankles because of traumatic injury or recurrent spontaneous joint bleeds during childhood and/or adolescence. Paradoxically, the success of treatment has led to a lack of awareness in some young people of the long-term consequences of nonadherence to treatment as well as social isolation ${ }^{[16]}$ The need to address this is reinforced by the 2014 Public Health England policy, which stated that: "There is a lot of evidence that the social element behind physical activity aids enjoyment. It also encourages people to stick at it" ${ }^{[17]}$.

For all the known benefits of physical activity, there is anecdotal evidence among the patient community to suggest that those living with haemophilia may be more averse to engaging in physical activity, due to fears of causing bleeding, joint pain and (further) joint damage.

\section{METHODOLOGY}

A series of qualitative interviews were conducted with young men with haemophilia of differing severity, to explore and identify reasons why young men show risk-averse behaviour towards exercise and physical activity. Participants, who all attended comprehensive care centres in London, were approached by the authors through their personal contact networks. After providing verbal consent, they were asked a series of open-ended questions during a telephone interview conducted by the authors, who together had formulated the interview schedule. These questions were designed to prompt discussion and capture opinions relating to participants' physical activity and gym membership/use, and the degree to which their haemophilia impacts on both (see Table 1). The interviews were recorded, transcribed verbatim and thematically analysed to identify key themes relating to attitudes towards physical activity. Direct quotes are used with consent. To protect anonymity, participants are known only by study number (P1, etc.). Ethical approval was not necessary using the Health Research Authority (HRA) decision tool application ${ }^{[18]}$ as the project did not include randomisation, change treatment/care or promote generalisable findings.

\section{RESULTS}

Ten participants aged $17-25$ years with mild, moderate or severe haemophilia A or B participated in the study (see Table 2). Each participant was interviewed once, 
1. General information:

- Age

- Occupation

- Type/severity of haemophilia

- Inhibitor

2. How active are you? What sort of sports or activities do you take part in?

3. Have you suffered any sports/activity-related injuries in the past?

4. Do these injuries still have an effect on your activity level today/currently?

5. Do you or have you been a member of a gym? How frequently did/do you attend?

- Prompt to expand on reasons for attendance/non-attendance

6. Is your haemophilia a consideration when you plan to exercise at the gym or engage in a sport?

- Prompt if necessary: Do you feel like your haemophilia may have dissuaded you from engaging in certain activities, either gym work or sport?

7. Do you feel comfortable and confident using a gym?

- Prompt: Is this something you would like to do more of?

Possibly expand on specific gym areas, e.g. machines, free weights, classes etc

8. Do you think attending a gym regularly would be of particular benefit to people with haemophilia?

9. Have you ever considered working out with a personal trainer?

[If yes, see how regularly, if maintained, etc. If no, prompt for reasons why not - preferably beyond cost]

10. Would a personal training programme tailored specifically to individuals with haemophilia, focused on gym-based activities, be of interest to you?

- Why/why not? What concerns would you have?

with an average interview recording time of 35 minutes (range 30-45 minutes). The themes identified from the interviews were: preferred activity, self-motivation, financial implications, sports injury, and the role of physiotherapy for musculoskeletal health.

\section{Preferred activity}

The extent to which the participants engaged in daily activity and their range of preferred activities varied widely. Five of the participants reported being 'very physically active', three reported 'moderate physical activity', and two did 'very little activity'. Four reported attending a gym and described themselves as having 'fairly high' to 'high confidence' in using a gym, whilst a further four reported being 'not gym confident'.

\section{Self-motivation}

Seven participants described themselves as highly or moderately motivated to engage in physical activity and exercise. In the case of Participant 3, weight loss was the main motivation to become more physically active:

"I got weighed at the [haemophilia] centre and I was like, 'OK, I'm not happy with that, let's go back to the gym.'" [P3]
For others, social motivations were revealed, such as wanting to get fit ahead of a summer holiday with a group of friends:

"I'm just really fed up of being unfit and my friends were saying the same." [P4]

Individuals with low levels of motivation described themselves as being 'lazy':

"When I was younger, I was doing martial arts, football, swimming... I was like any other ordinary kid. As I've got older, I get more lazy." [P5]

Despite a close relationship with his clinical team and physiotherapist, P5 said he "rarely did the exercises recommended to him by his physiotherapist". P10, who described himself as highly motivated, said he was "trying to build muscle and strength but felt he was often too lazy".

The participants also raised the issue of confidence in undertaking weight-bearing exercise, with one participant revealing that he was "more confident to try these types of exercises with [his] physio at the centre," rather than on his own. There was some anxiety around exercising due to fear of pain or injury: 
"I would like to do more weight-bearing exercises. But I just know with my elbows that I can't... I know what my limits are in terms of causing haemophilia arthropathy pain. It's finding out what I can do without either causing bleeds or just causing my joints to ache." [P6]

\section{Financial aspects}

All participants had exposure to personal trainers (PT) at their gyms and recognised the potential benefits of working with a PT. The importance of an introduction to training equipment was expressed by the participants:

\section{"When you start doing some exercises, you always want to do them correctly and... you can only do them correctly when you're under someone's supervision... who is a professional in that area." [P2]}

"When you work out a lot, sometimes it's hard to recognise if you're doing something a little wrong. With a personal trainer you can correct your activity." [P8]

Some participants recognised the potential benefits of working with a PT but had concerns about their own motivation:

"I think it would benefit me but I would need to be really motivated and supported to do it." [P5]

All participants felt that working with a PT would be too expensive for them and four had not used a PT due to cost alone. One frequent gym user had previously used a trainer to devise his own programme but added:

"I don't really want to spend that much [on a $P T]$ and I know roughly what I'm doing, so I can build on what they tell me." [P1]

Injury

The majority of participants reported that they had sustained sports-related injuries, which they acknowledged was likely worsened in severity and recovery due to their haemophilia. They appeared to be aware of their limitations, especially those with severe haemophilia who understood the need for prophylaxis prior to physical activity:

"Without the concentrates, you wouldn't be able to go to the gym, but because the concentrates are available... it's a bit irresponsible not to do exercise... which leads to improved body functioning and reduced bleeds." [P2]

\section{Physiotherapy}

The participants reported that physiotherapists are often their first point of contact for advice and support regarding safe physical activity:

"You do hear repetitively from physios that the healthier you are or the more you do with your joints, the more protected they are from bleeding." [P6]

The participants reported a view that most physiotherapists provided only a 'prescription' of stretches and exercises, usually focused on specific musculoskeletal problems rather than on endpoints, such as being able to lift weights, run a $10 \mathrm{~K}$ race and participate in sport without thinking about haemophilia likely in their everyday lives. All participants recognised the benefits of physical activity and expressed an interest in wanting to do more. Most said they had been encouraged to do so by their physiotherapists in order to augment the efficacy of their treatment. Participants acknowledged that improving fitness and building muscle would lead to better outcomes:

"For me... it would probably be better if I built up muscle around my joints." [P5]

"The ultimate goal is to strengthen the joints and muscles... there is a huge benefit... you can have prophylaxis, you can have amazing treatment but as long as your joints, tendons and muscles are weak, your joints will be like jelly and they will keep bleeding." [P2]

\section{DISCUSSION}

We have shown that this group of young men with haemophilia, mostly treated with regular prophylaxis, already participate in sporting activity, including some that were previously considered 'risky' (e.g. martial arts, cricket) despite target joint(s) and/or the risk of injury. Despite this, there is evidence to suggest that they are not reaching their full potential for physical activity, due to lack of knowledge and fears around potential bleeding, joint pain and exacerbating previous injuries. This suggests that there is a need to establish a more tailored approach to exercise, focusing on identifying 
and addressing an individual's underlying medical and psychological issues that may impact on their personal motivation

Gue et al. describe a new model of haemophilia care that supports patient autonomy in decisions related to managing their haemophilia, by de-emphasising treatment adherence as the primary goal and focusing on a healthcare plan that is customised by the patient and aligned with their priorities ${ }^{[19]}$. To support physical activity, this could include personalised exercise plans developed between the person with haemophilia and his clinical team and include motivational interviewing and goal setting ${ }^{[20]}$

Our results indicated varied motivation to engage in physical activity, including weight loss and getting fit for the summer. Overweight and obesity in people with haemophilia is recognised as a double-edged sword with the impact of haemophilia (bleed-related risks) negatively influencing the ability and intensity of exercise ${ }^{[21]}$. Individualised education and "tailoring engagement in physical activity to avoid the risk of traumatic bleeding" is suggested by Wilding et al. ${ }^{[22]}$. This would involve both a physiotherapy assessment to examine musculoskeletal health and personalised training programmes developed with a PT. Participants saw the potential benefits of working with a PT to become more confident and competent in a gym setting, acknowledging the importance of feeling selfassured and being familiar with using equipment and practicing various exercises safely and effectively in order to reduce the risk of injury.

Participants commented that PTs were expensive, and for some this prevented them from using this expertise, which may lead to injury. Several participants recounted joint or muscle bleeds following sporting trauma (Table 2) despite pre-sport prophylaxis, some of which caused lasting impact. Participant 8 reported a target knee joint that started ten years previously following a football injury. Physiotherapists within the haemophilia team are used as the first point of contact after bleeds occur for rehabilitation but are also reported to encourage sport and activity, including gym use, to improve fitness and wellbeing.

\section{CONCLUSION}

We have shown that young men with haemophilia of differing severity are keen to use the gym as part of their individualised personal fitness/training regimens, yet many healthcare professionals continue to have concerns about safety. This is an area that warrants further research.

\section{ACKNOWLEDGEMENTS}

We would like to thank the participants who took part in these interviews.

This study was funded by Haemnet.

Informed consent has been obtained by the participants in the study reported in this paper.

\section{ORCID}

Luke Pembroke iD https://orcid.org/0000-0002-2024-6898 Laurence Woollard (iD https://orcid.org/0000-0002-1494-3427

\section{REFERENCES}

1. Weigle N, Carson BR. Physical activity and the hemophiliac: yes or no? Am Correct Ther J 1975; 29: 197-205.

2. Fischer K, Ljung R. Primary prophylaxis in haemophilia care: Guideline update 2016. Blood Cells Mol Dis 2017; 67: 81-85. doi:10.1016/j.bcmd.2017.02.004

3. Negrier C, Seuser A, Forsyth A, et al. The benefits of exercise for patients with haemophilia and recommendations for safe and effective physical activity. Haemophilia 2013; 19(4): 48798. doi: 10.1111/hae.12118.

4. Pelletier JR, Findley TW, Gemma SA. Isometric exercise for an individual with hemophilic arthropathy. Phys Ther 1987; 67(9): 1359-64. doi: 10.1093/ptj/67.9.1359.

5. von Mackensen S, Czepa D, Herbsleb M, Hilberg T. Development and validation of a new questionnaire for the assessment of subjective physical performance in adult patients with haemophilia - the HEP-Test-Q. Haemophilia 2010; 16(1): 170-8. doi: 10.1111/j.1365-2516.2009.02112.x.

6. Broderick CR, Herbert RD, Latimer J, et al. The effect of an exercise intervention on aerobic fitness, strength and quality of life in children with haemophilia. BMC Blood Disorder 2006; 6: 2. doi: 10.1186/1471-2326-6-2.

7. Herbsleb M, Hilberg T. Maximal and submaximal endurance performance in adults with severe haemophilia. Haemophilia 2009; 15(1): 114-21. doi: 10.1111/j.1365-2516.2008.01860.x.

8. Khair K, Littley A, Will A, von Mackensen S. The impact of sport on children with haemophilia. Haemophilia 2012; 18; 898-90. doi: 10.1111/j.1365-2516.2012.02857.x.

9. Czepa D, von Mackensen S, Hilberg T. Haemophilia \& Exercise Project (HEP): the impact of 1-year sports therapy programme on physical performance in adult haemophilia patients. Haemophilia 2013; 19(2): 194-9. doi: 10.1111/hae.12031.

10. von Mackensen S, Harrington $C$, Tuddenham E, et al. The impact of sport on health status, psychological well-being and physical performance of adults with haemophilia. Haemophilia 2016; 22; 521-30. doi: 10.1111/hae.12912.

11. Runkel B, Von Mackensen S, Hilberg T. RCT - subjective physical performance and quality of life after a 6 -month programmed sports therapy (PST) in patients with haemophilia. Haemophilia 2017; 23(1): 144-51. doi: 10.1111/hae.13079.

12. Souza JC, Simoes HG, Campbell CS, et al. Haemophilia and exercise. Int J Sports Med 2012; 33(2): 83-8. doi: 10.1055/s0031-1286292

13. Strike K, Mulder K, Michael R. Exercise for haemophilia Cochrane Database Syst Rev 2016; 12: CD011180. doi: 10.1002/14651858.CD011180.pub2. 
14. Tiktinsky R, Falk B, Heim M, Martinovitz U. The effect of resistance training on the frequency of bleeding in haemophilia patients: a pilot study. Haemophilia 2002; 8(1): 22-7. doi: 10.1046/j.1365-2516.2002.00575.x.

15. Tiktinsky R, Kenet G, Dvir Z, et al. Physical activity participation and bleeding characteristics in young patients with severe haemophilia. Haemophilia 2009; 15(3): 695-700. doi: 10.1111/j.1365-2516.2009.01990.x.

16. Khair K, Gibson F, Meerabeau L. 'I can always rely on them': the importance of social support for boys with haemophilia. J Haem Pract 2013; 1(1) 10-16. doi: 10.17225/jhp.0005.

17. Public Health England. Everybody Active, Every Day: Implementation and evidence guide. 2014. Available from https://assets.publishing.service.gov.uk/government/uploads/ system/uploads/attachment_data/file/353385/Everybody_ Active__Every_Day_Implementation__Evidence_Guide_ CONSULTATION_VERSION.pdf (accessed 20 May 2020).

18. UKRI Medical Research Council; NHS Health Research Authority. HRA decision tools: Is my study research? http://www.hradecisiontools.org.uk/research/ (accessed 20 July 2017).

19. Gue D, Squire $S$, Mclntosh $K$, et al. Joining the patient on the path to customized prophylaxis: one hemophilia team explores the tools of engagement. J Multidiscip Healthc 2015; 8: 527-34. doi:10.2147/JMDH.S93579.
20. Roberts JC, Lattimore S, Recht M, et al. Goal Attainment Scaling for haemophilia (GAS-Hēm): testing the feasibility of a new patient-centric outcome measure in people with haemophilia. Haemophilia 2018; 24(4): e199-e206. doi:10.1111/hae.13454.

21. Kahan S, Cuker A, Kushner RF, et al. Prevalence and impact of obesity in people with haemophilia: Review of literature and expert discussion around implementing weight management guidelines. Haemophilia 2017; 23(6): 812-20. doi:10.1111/ hae.13291.

22. Wilding J, Zourikian N, Di Minno M, et al. Obesity in the global haemophilia population: prevalence, implications and expert opinions for weight management. Obes Rev 2018; 19(11): 1569-84. doi:10.1111/obr.12746.

HOW TO CITE THIS ARTICLE:

Pembroke L, Woollard L. Perceptions and attitudes

towards gym use and physical activity in young men with haemophilia. J Haem Pract 2020; 7(1): 85-91. https://doi. org/10.17225/jhp00164.

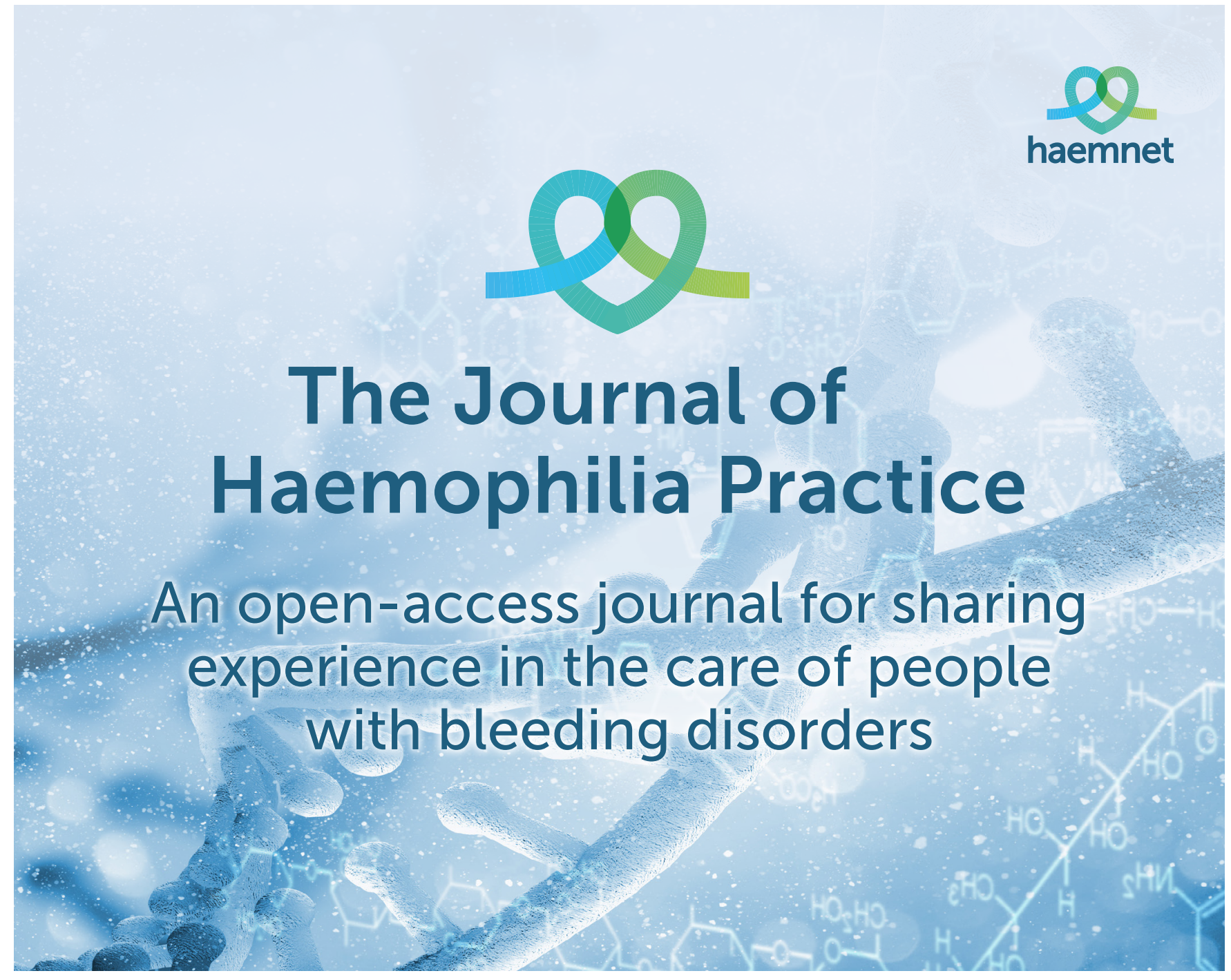




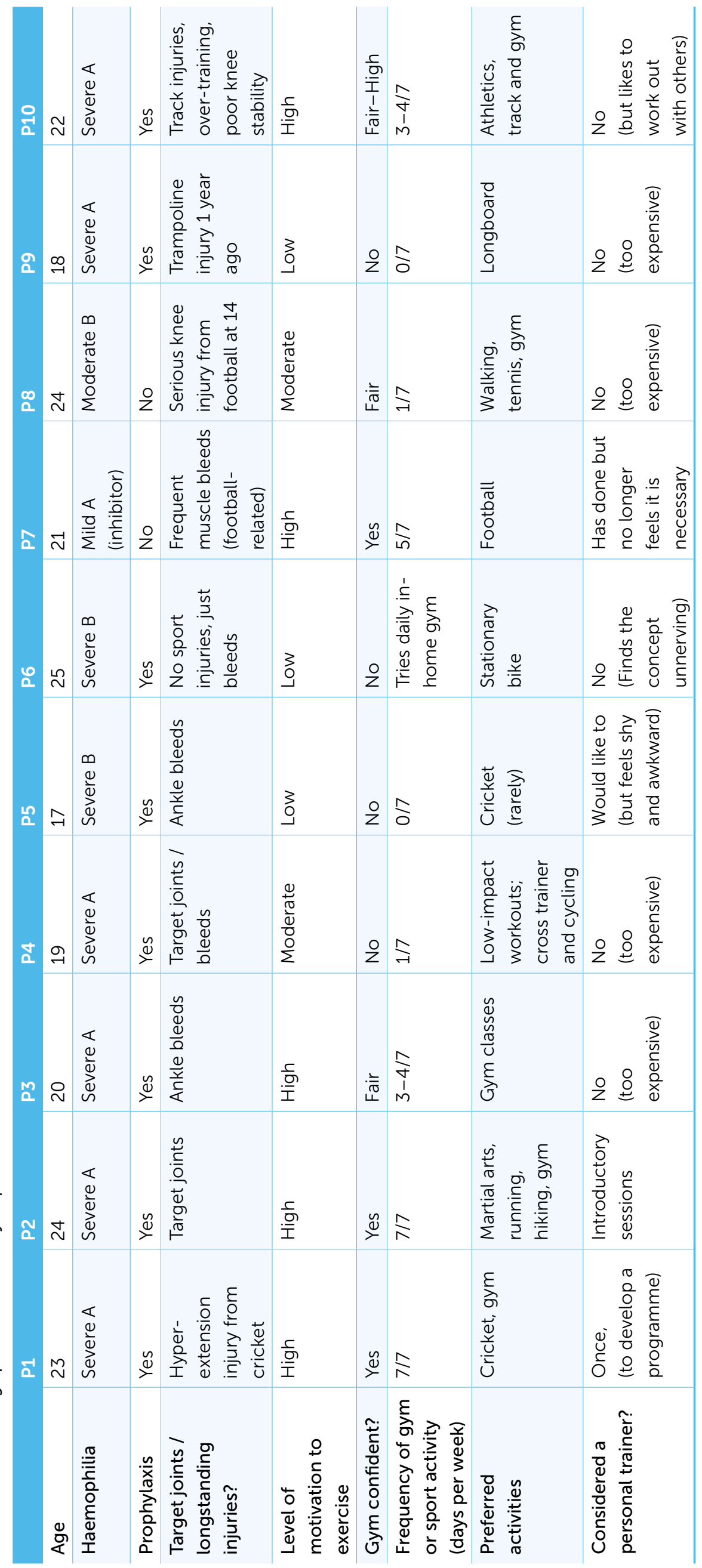

